\title{
IMPORTÂNCIA DA AVALIAÇÃO GENÉTICO-CLÍNICA NA HIDROCEFALIA
}

\author{
Marta Wey-Vieira ${ }^{1}$, Denise Pontes Cavalcanti², Vera Lúcia Gil Silva Lopes $^{2}$
}

\begin{abstract}
RESUM O - Os objetivos deste estudo foram caracterizar a presença de possíveis quadros de etiologia genética entre portadores de hidrocefalia congênita de etiologia não anteriormente esclarecida e confirmar aqueles com etiologia identificada previamente. A casuística compôs-se de 16 pacientes portadores de hidrocefalia congênita. 0 protocolo de investigação incluiu anamnese, investigação de história familial, exame clínico-dismorfológico, tomografia computadorizada ou ressonância magnética de sistema nervoso central, radiografia vertebral simples, cariótipo e estudo dismorfológico. Para análise dos resultados, a casuística foi dividida em dois grupos. 0 Grupo I (3M :6F) caracterizado por indivíduos com hidrocefalia e sinais clínicos inespecíficos; 0 Grupo II (7M), em que os indivíduos apresentavam hidrocefalia congênita e sinais sugestivos do espectro da doença L1. Orientação genética específica foi possível em 11 casos. Os resultados demonstram a heterogeneidade etiológica envolvida na hidrocefalia, evidenciando a necessidade de avaliação clínico-dismorfológica como instrumento complementar na investigação dessa condição clínica.
\end{abstract}

PALAVRAS-CHAVE: hidrocefalia, dismorfologia, aconselhamento genético.

\begin{abstract}
Importance of the clinical genetics evaluation on hydrocephalus
ABSTRACT - The aim of this study was to characterize the possibility of genetic etiology in a group of individuals with congenital hydrocephalus in which the etiology was indeterminate and to confirm that earlier diagnosed. The casuistry was composed by 16 individuals with congenital hydrocephalus. Investigation protocol included anamnesis, familial investigation, physical examination, computerized tomography or magnetic resonance image of head, vertebral column X-ray, karyotype and dysmorphological study. Results were analyzed in two groups. In Group I (3M:9F) was composed by hydrocephalus associated with unspecific signs. Group II (7 males) had findings of epectrum of L1 disease. Genetic counseling could be offered in 11 cases. These results demonstrate the great etiological heterogeneity of congenital hydrocephalus and reinforce the importance of dysmphology evaluation as an important complementary investigation.
\end{abstract}

KEY WORDS: hydrocephalus, dysmorphology, geneticounseling.

A hidrocefalia congênita é definida como condição clínica decorrente de distúrbio da circulação do líquido céfaloraquidiano (LCR) resultando em dilatação ventricular progressiva. Trata-se, portanto, da expressão clínica de algum defeito primário, na maioria das vezes obstrutivo, com significativa heterogeneidade etiológica ${ }^{1}$. Trata-se de condição clínica prevalente em diferentes populações, referida como 0,5 a 2,5/1000 nascidos vivos ${ }^{2}$. Em estudo de revisã $0^{3}$, foi encontrada incidência de 0,4 a 0,8/1000 nascimentos, entre nativivos e natimortos. As incidências brasileiras registradas pelo Estudo Colaborativo Latino Americano de Malformações Congênitas (ECLAMC), incluindo nativivos e natimortos com $500 \mathrm{~g}$ ou mais, variaram de $6,2 / 10000$ a 50,3/10000 nascimentos, de acordo com a região $0^{1}$. Nos últimos anos, a incidência da hidrocefalia congênita tem mostrado tendência a aumento no
ECLAMC e, em especial em hospitais universitários como é o caso da Maternidade do CAISM (Centro de Atenção Integral à Saúde da M ulher/UNICAM P). Essa ascensão está fortemente associada ao diagnóstico pré-natal ${ }^{4}$.

Há diversas condições etiológicas determinantes da hidrocefalia congênita. Incluem-se as determinadas por fatores ambientais (teratogênese), associadas a outros defeitos congênitos, genéticos ou não, e as geneticamente determinadas. Considerando a idade de ocorrência e de manifestação para diagnóstico diferencial, merece menção, também, a etiologia perinatal traumática. Do ponto de vista genético-clínico, a hidrocefalia pode ser classificada em isolada, ou associada a outros defeitos congênitos. As isoladas, em geral, podem ser resultantes ou estar associadas a malformações do, sistema nervoso central (SNC), ou a infecções congênitas. Destaca-se, ainda, a hidrocefalia

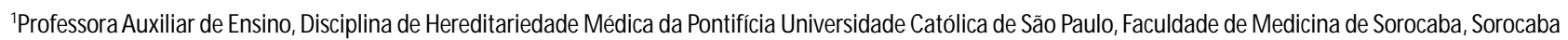
SP, Brasil; ${ }^{2}$ Professor Doutor, Departamento de Genética M édica, Faculdade de Ciências Médicas Universidade Estadual de Campinas (FCM -UNICAM P), Campinas SP, Brasil.

Recebido 6 Novembro 2003, recebido na forma final 29 Dezembro 2003. Aceito 23 Janeiro 2004.

Dra. Vera Lúcia Gil da Silva Lopes - Departamento de Genética Médica FCM / UNICAMP - Caixa Postal 6111 - $13083-971$ Campinas SP - Brasil. E-mail: vlopes@fcm.unicamp.br. 
decorrente de defeitos de fechamento de tubo neural, no qual a prevenção com ácido fólico tem boa eficácia 5 . Dentre as hidrocefalias associadas a outros defeitos congênitos, pode-se encontrar casos sem definição clínico-etiológica constituindo os polimalformados verdadeiros ou aqueles com uma síndrome dismórfica definida ${ }^{1}$.

Dentre as hidrocefalias de etiologia genética, encontramse as determinadas por anomalias cromossômicas, herança monogênica, com padrões de transmissão do tipo autossômico dominante, autossômico recessivo e ligado ao X recessivo ${ }^{6}$. Nessa última categoria, destaca-se o Espectro da Doença L1, a qual, após estudo de ligação, incluiria as três condições clínicas ligadas ao $X^{7}$, a saber: paraplegia espástica ligada ao X (OMIM 3129008), síndrome MASA (OMIM 3033508) e hidrocefalia ligada ao $X$ com estenose do aqueduto de Sylvius (OM IM 3070008). Estas seriam, na verdade, manifestações clínicas variáveis da mesma mutação em Xq28, ou, ainda, poderiam representar mutações diferentes no mesmo locus. Um segundo locus desta condição em Xq27.3 foi aventado9.

No espectro da doença L1, o quadro clínico é bastante variável, sendo descrito desde hidrocefalia grave, resultando em morte perinatal, até retardo mental leve como anomalia única ${ }^{10}$. Os principais sinais associados descritos são hidrocefalia, geralmente por estenose de aqueduto de Sylvius, retardamento mental, polegares aduzidos, paraplegia espástica, baixa estatura, lordose lombar acentuada e intensificação dos reflexos tendinosos profundos nos membros inferiores, sendo verificado, também, significativa variabilidade intra e extra-familial. Existem, também, descrições de expressão sutil em mulheres portadoras do gene, caracterizada por inteligência limítrofe e (ou) polegares aduzidos ${ }^{1,3,11-13}$ Assim, no sexo feminino, existe a necessidade de exame clínico cuidadoso, dando especial atenção ao status mental, fenótipo e sintomas neurológicos. Outro dado interessante sobre o espectro da doença L1 é que, a partir da correlação entre o sítio de mutação e o fenótipo, foi verificado que diferentes pacientes com mutações no mesmo domínio na proteína L1CAM apresentavam fenótipos distintos, tanto do ponto de vista dismorfológico quanto de gravidade ${ }^{10}$.

Nesse artigo, descrevem-se os resultados obtidos após avaliação genético-clínica de portadores de hidrocefalia congênita previamente avaliados e para os quais as etiologias mais comuns de hidrocefalia já haviam sido afastadas. Este estudo teve como objetivo caracterizar a presença de possíveis quadros de etiologia genética entre portadores de hidrocefalia congênita de etiologia não anteriormente esclarecida e de confirmar aqueles com uma etiologia já identificada previamente.

\section{MÉTODO}

Foram incluídos 16 pacientes portadores de hidrocefalia congênita encaminhados para investigação genético-clínica, sendo 8 casos novos e 8 reavaliados após consulta genético clínica no mesmo serviço, sendo 10 do gênero masculino e 6 do feminino; a idade variou de 4 dias de vida a 13 anos e 4 meses.

Os critérios de inclusão foram: hidrocefalia congênita ou de desenvolvimento pós-natal precoce, ausência de defeito de fechamento de tubo neural, ausência de anomalias oftalmológicas, ausência de infecção congênita comprovado por meio da investigação de história gestacional e realização de sorologias para sífilis, rubéola, citomegalovírus, toxoplasmose e herpes, não sendo incluídos os pacientes com imunoglobulina $M$ positiva; além de exame de cariótipo normal em técnica de banda $\mathrm{G}$ com resolução de pelo menos 450 bandas por lote haplóide.

Foram excluídos os indivíduos com história reconhecida de anóxia perinatal grave ou qualquer das investigações acima anormais.

Todos os indivíduos participantes foram examinados pessoalmente pelos autores, sendo as informações obtidas diretamente dos responsáveis pelo paciente e transcritas para um protocolo padrão de anamnese, incluindo a confecção de heredogramas conforme estabelecido ${ }^{13}$; além da realização de exame dismorfológico ${ }^{14}$. No estudo genético-clínico dos casos foi realizada revisão de literatura específica para cada caso.

A investigação complementar constou de exames de imagem do SNC (ultra-som transfontanelar, tomografia computadorizada de crânio, e/ou ressonância magnética de crânio), exames radiológicos de coluna vertebral (cervical, torácica e lombo-sacral) e mãos. Outros exames complementares foram solicitados, quando indicados.

Esse estudo foi aprovado pelo Comitê de Ética da Faculdade de Ciências M édicas/UNICAMP (no.463/2002).

\section{RESULTADOS}

Os indivíduos participantes foram divididos em dois grupos: Grupo l: composto por 9 indivíduos (3 masculinos e 6 femininos) com hidrocefalia sem outros sinais sugestivos do espectro da doença L1(casos 1 a 9); Grupo II: composto por 7 indivíduos do gênero masculino com hidrocefalia e sinais sugestivos do espectro da doença L1 (casos 10 a 16).

No Grupo I, em três indivíduos existiram ocorrências de fatores pré-natais inespecíficos (febre, infecção urinária); dois indivíduos apresentaram sinais sugestivos de associação VACTERL-H, porém não suficientes para conclusão diagnóstica; dois afetados de gêneros diferentes em uma mesma irmandade, cujos genitores não são consangüíneos; em dois indivíduos não foram detectados elementos suficientes para aventar uma hipótese diagnóstica.

$\mathrm{Na}$ avaliação clínico-dismorfológica da casuística, observase importante heterogeneidade. Nos dois indivíduos que apresentaram, além de hidrocefalia, sinais que podem sugerir a associação VACTERL-H foi observado em um deles, do gênero feminino, atresia de esôfago, cardiopatia e anomalia vertebral, com fusão dos corpos vertebrais de L5 e S1 e de S3 e S4, além da última vértebra mais alargada. 0 outro, do gênero masculino, além da hidrocefalia, apresentava anomalia renal (agenesia renal à esquerda) e vertebral, com fusão de C6-C7, espinha bífida em T1,T2 e S1, costela cervical à esquerda, irregularidade de alguns corpos vertebrais (últimas vértebras torácicas e primeiras vértebras lombares), além de hemivértebra em 
T11 e 11 costelas à esquerda.

Os dois indivíduos da mesma irmandade apresentavam sinais clínicos discretos como macrocefalia com hidrocefalia e fronte abaulada, além do atraso de desenvolvimento neuropsicomotor.

O Grupo II caracterizou-se por sete indivíduos com sinais sugestivos do espectro da doença $L 1$, sendo que em quatro casos, a história familial foi positiva (Tabela 1).

\section{DISCUSSÃO}

Grupo 1

Sobre os antecedentes gestacionais da amostra analisada, observa-se que, em três indivíduos, há relato de febre ou infecção urinária. Nesses, o período da intercorrência gestacional foi anterior ao da detecção da hidrocefalia, porém não se pode afirmar que exista correlação entre esses dois fatos.

A despeito de diferentes etiologias, a febre materna age como importante agente teratogênico. As manifestações mais freqüentes seriam deficiência mental grave, convulsões, hipotonia, microftalmia, hipoplasia da face média, defeitos de fechamento do tubo neural, hipotonia e aumento dos reflexos profundos, além de outros dismorfismos faciais ${ }^{15-17}$. Muitos casos de retardo mental idiopático na espécie humana, poderiam ser relacionados à febre materna durante um período gestacional precoce ${ }^{18}$. A pesar desses achados, estudos de revisão sobre hipertermia materna gestacional mencionam a hidrocefalia como sinal secundário aos defeitos de fechamento de tubo neural.

Levando-se em conta o quadro de infecção de trato urinário mencionado na história gestacional de dois indivíduos, poderia ser aventada a hipótese de uma relação entre este evento e o quadro clínico apresentado por tais pacientes. A associação de infecção urinária gestacional com paralisia cerebral já foi mencionada ${ }^{19}$. Outros estudos de coorte realizados ${ }^{20,221}$, relacionam infecção urinária no 30 trimestre com retardo mental, retardo de desenvolvimento pós-natal e morte fetal. Apesar desses achados, não foi referida a presença de hidrocefalia. Por outro lado, a infecção de trato urinário constitui uma das intercorrências gestacionais mais freqüentes, na qual a presença de hidrocefalia não costuma estar associada.

0 acrônimo VACTERL significa anomalia vertebral, atresia anal, malformações cardíacas, fístula traqueoesofágica, anomalia renal e anomalia de membro (limb), que em geral apresenta baixo risco e recorrência ${ }^{22}$. Contudo, alguns autores ${ }^{23,24}$ relataram a ocorrência familial de VACTERL com hidrocefalia (VACTERL-H). Inicialmente, foi considerada a possibilidade de herança autossômica recessiva ${ }^{25}$, porém, em 1996, foi descrita a ocorrência em dois irmãos do gênero masculino, sugerindo a possibilidade de herança recessiva ligada ao $\mathrm{X}^{26}$. Tal possibilidade foi corroborada pelo relato de uma família com quatro meninos com hidrocefalia, anormalidade de arco radial, anomalia renal, atresia anal, pênis hipoplásico e testículos anormais, além de cardiopatia ${ }^{27}$. Nesta família, uma prima materna apresentava fístula traqueoesofágica, representando manifestação parcial de uma mulher portadora.

Nos dois indivíduos em questão, considerando que ainda faltam elementos para conclusão diagnóstica e que não existe história familial ou gestacional relevante, não foi possível uma conclusão. Assim, do ponto de vista clínico, o mais adequado seria a manutenção do seguimento ambulatorial, aguardando a evolução dos pacientes. Do mesmo modo, 0 aconselhamento genético não pôde ser realizado com precisão.

Outros dois afetados, que são irmãos, de gêneros diferentes,

Tabela 1. Principais dados referentes à avaliação genético-clínica do Grupo II.

\begin{tabular}{cll}
\hline Indivíduo & Principais sinais clínicos & Principais dados da história \\
10 & Macrocefalia, baixa estatura & Mãe e irmã com deficiência mental \\
11 & $\begin{array}{l}\text { Macrocefalia, nistagmo, alteração da coordenação } \\
\text { baixa estatura, polegares adutos }\end{array}$ & Tio materno e tio-avô materno com hidrocefalia, \\
12 & $\begin{array}{l}\text { Braquicefalia, epicanto, braquidactilia, baixa estatura, } \\
\text { alteração da coordenação }\end{array}$ & Não há história familial digna de nota \\
13 & $\begin{array}{l}\text { Macroceflia, polegares adutos, nistagmo, alteração da } \\
\text { coordenação }\end{array}$ & Não há história familial digna de nota \\
15 & $\begin{array}{l}\text { Macrocefalia, epicanto, nistagmo, alteração da } \\
\text { coordenação, polegares adutos }\end{array}$ & $\begin{array}{l}\text { Meio-irmão materno que faleceu com } \\
\text { hidrocefalia e doença de Hirschsprung }\end{array}$ \\
16 & Fronte abaulada, nistagmo, alteração da coordenação, & Relato de vários casos de hidrocefalia pelo lado \\
baixa estatura, polegares adutos & Macrocefalia, baixa estatura & Sem história familial \\
\hline
\end{tabular}




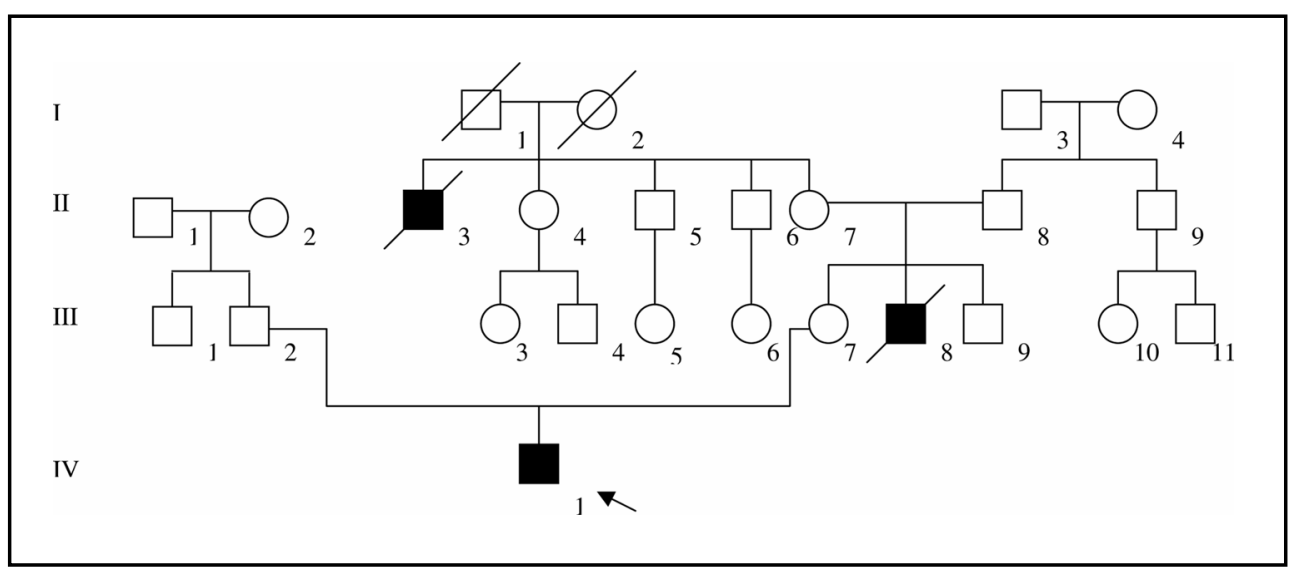

Fig 1. Heredograma do caso 11 evidenciando o padrão de transmissão recessivo ligado ao $X$.

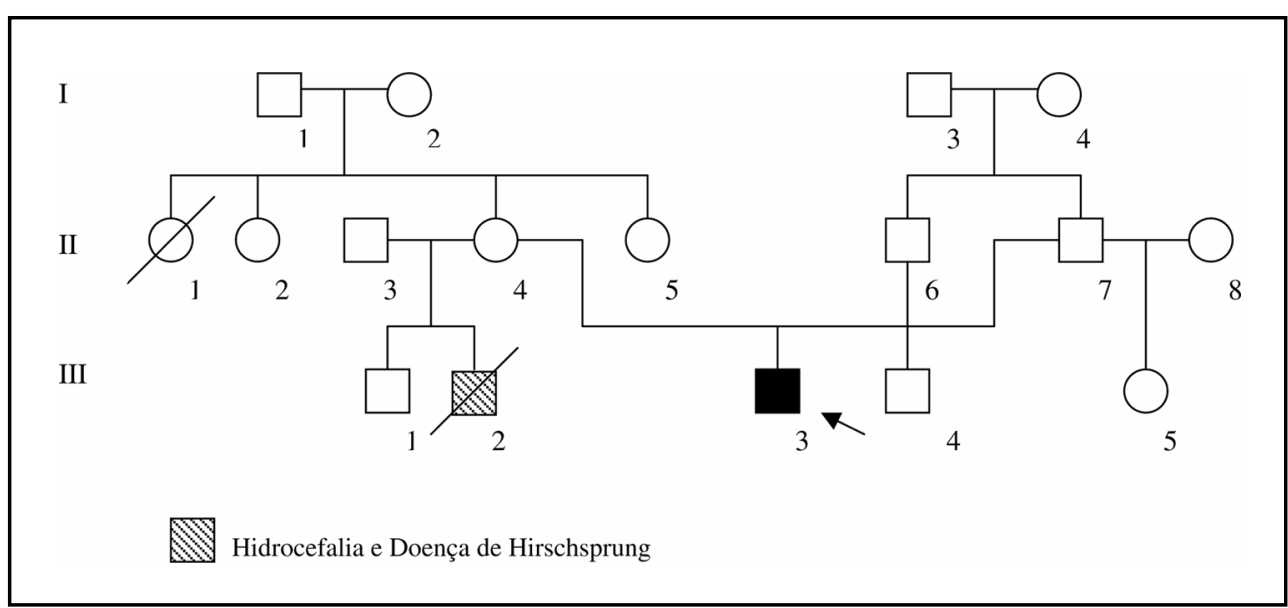

Fig 2. Heredograma do caso 14: observar a presença de meio-irmão com hidrocefalia e Doença de Hirshprung, sugerindo o espectro da Doença L1.

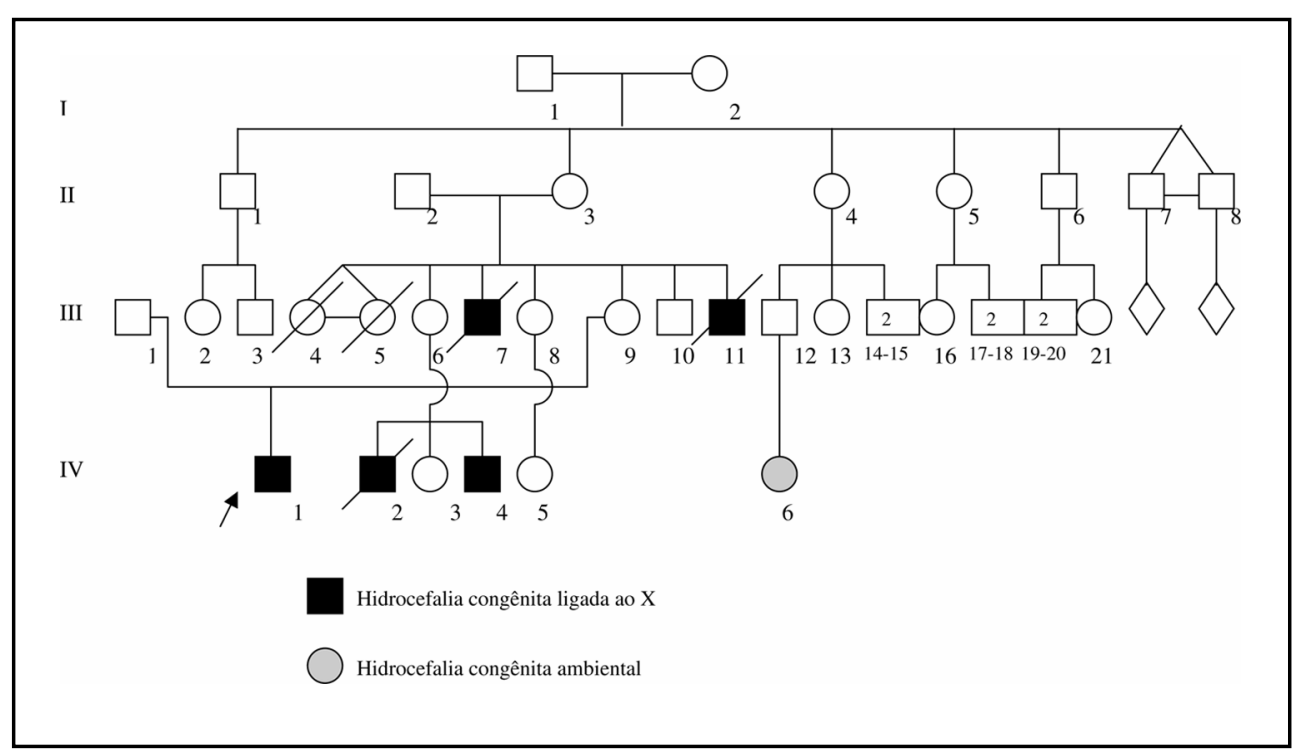

Fig 3. Heredograma do caso 15: recorrência de hidrocefalia congênita caracterizando transmissão recessiva ligada ao X. 
apresentam quadro clínico muito semelhante, sugerindo a possibilidade de herança autossômica recessiva ou, ainda, recessiva ligada ao $X$ com expressão em heterozigotas. Em 1982, foi questionada a existência de uma forma autossômica recessiva de hidrocefalia (Optiz, Comunicação Pessoal. In OM IM 236600). Tal fato teve origem na observação de somente uma irmandade na qual irmãos de sexos diferentes apresentavam hidrocefalia. Em amplo estudo ${ }^{28}$ analisando a ocorrência de hidrocefalia no período de vinte anos, observouse a possibilidade de herança autossômica recessiva em apenas duas famílias dentre as 91 estudadas, 0 que poderia reforçar a opinião de Optiz. De todo modo, esse fato não afastaria a possibilidade de um gene autossômico recessivo de baixa freqüência, ou ainda, de um modelo de transmissão recessivo ligado ao X com expressão em heterozigotos.

Herança autossômica dominante com expressividade variável (HADV) poderia vir a ser investigada nos genitores ${ }^{29}$. A possibilidade da existência de um gene contíguo situado no cromossomo 8q12.2-q21.2, que estaria relacionado com hidrocefalia, síndrome BOR (Brânquio-0to-Renal) ${ }^{30}$ e síndrome Duane (hidrocefalia e agenesia de músculo trapézio). Assim, na família da presente casuística, seria adequado o exame clínico cuidadoso dos genitores, a fim de verificar a possibilidade desses achados, o que não foi possível. Quanto ao aconselhamento genético, afastando a hipótese de HADV, não está excluída a possibilidade de herança autossômica recessiva, sendo o aconselhamento genético realizado nesse sentido.

\section{Grupo II}

Composto por sete indivíduos, o Grupo II incluiu os que apresentaram quadro clínico sugestivo do espectro da doença L1. Destes, além dos sinais relacionados ao quadro de hidrocefalia, destacam-se a baixa estatura, polegares adutos e alteração da coordenação, como sinais sugestivos desta condição clínica. Nos casos 10, 11, 14 e 15 existem fortes indícios de herança ligada ao $X$, tendo em vista a história familial (Figs 1, 2 e 3).

$\mathrm{Na}$ avaliação do indivíduo 12 observa-se a presença de sinais dismórficos pouco freqüentes no espectro da doença $L 1$, como a braquicefalia, as pregas epicânticas, a braquidactilia e sindactilia, além da cardiopatia. Tais sinais sugeriram, inicialmente, a possibilidade de uma cromossomopatia, descartada pelo cariótipo, que resultou normal. Contudo, este paciente apresenta ainda outros sinais dismórficos freqüentes no espectro da doença $L 1$, como a dilatação ventricular moderada, os polegares aduzidos, o comprometimento neurológico grave e as alterações de coluna vertebral. Nessa situação seria adequado o seguimento clínico do propósito e de sua família, para se possível, conclusão diagnóstica e a realização do estudo molecular do gene LICAM.
0 paciente 13 não apresenta história familial relevante; 0 que parece ser significativo neste caso é o quadro dismórfico, com a presença de sinais tipicamente associados ao espectro da doença L1, como, por exemplo, a macrocefalia com hidrocefalia, polegares aduzidos, alteração da coordenação e nistagmo.

No caso 14 nota-se o óbito de um meio-irmão materno com hidrocefalia e doença de Hirschsprung. Existe uma descrição de um menino com dois anos de idade com quadro da síndrome MASA e doença de Hirschsprung ${ }^{31}$. Mais recentemente, houve sugestão de que a isoforma não-neural da molécula L1 se expressa nas células da cripta intestinal ${ }^{32}$. Por sua vez, a hipótese de que a molécula L1CAM , por ser uma molécula de adesão celular, pode ser importante para possibilitar as células precursoras ganglionares povoarem o intestino e, portanto, uma mutação no gene L1CAM poderia provocar a aganglionose levando à doença de Hirschsprung ${ }^{32}$ a ser considerada nesse caso. Considerando essa hipótese, na família em questão, pode-se caracterizar o modo de transmissão ligado ao X.

No indivíduo 15 nota-se a ocorrência de vários casos de hidrocefalia, todos pelo lado materno, sugerindo a herança recessiva ligada ao $X$, compatível com o espectro da doença L1, que também é sugerido pelo seu quadro clínico.

Nos indivíduos do grupo II, com exceção do caso 16, 0 aconselhamento genético foi realizado como espectro da doença $L 1$, de etiologia recessiva ligada ao cromossomo $X$.

0 indivíduo 16 apresenta quadro clínico muito sutil, com comprometimento neurológico leve e sem história familial prévia, porém com imagem tomográfica sugestiva de estenose de aqueduto de Sylvius. Nessa situação, embora o quadro clínico seja frustro, e o paciente tenha sido mantido em seguimento clínico, o aconselhamento genético foi realizado considerando a hipótese do espectro da doença L1.

\section{CONCLUSÃO}

Trata-se, portanto, de uma casuística heterogênea. Nos casos em que houve envolvimento de fatores pré-natais, estes não podem ser relacionados com o quadro clínico. Assim, estudos epidemiológicos futuros poderiam verificar a real importância dos eventos pré-natais detectados nesta casuística na gênese das hidrocefalias.

Por outro lado, em 11 dos 16 indivíduos existiu alguma orientação específica referente ao aconselhamento genético de seus genitores, o que poderia beneficiar, também, outros indivíduos na família, quer estabelecendo risco significativo ou desprezível.

Assim, avaliação clínico-dismorfológica pode ser considerada como um instrumento complementar para investigação de casos de hidrocefalia, com o objetivo de se determinar a etiologia e promover um aconselhamento genético adequado 
aos genitores e, eventualmente aos demais familiares interessados. Além disso, o estudo molecular visando à identificação de mutações no gene L1CAM poderia ser de auxílio diagnóstico nos casos aqui investigados, permitindo, se detectadas mutações, aconselhamento genético específico e possibilitando diagnóstico pré-natal. Esse procedimento poderia contribuir, ainda, para o estabelecimento da expressão clínica parcial em heterozigotas.

Agradecimento - As autoras agradecem a colaboração das famílias que concordaram em participar deste estudo.

\section{REFERÊNCIAS}

1. Salomão M A. Estudo sobre hidrocefalias congênitas em um hospital universitário brasileiro. Dissertação de Mestrado, UNICAMP. Campinas, 2000.

2. Vintzileos AM, Ingardia CJ, Nochimson DJ. Congenital hydrocephalus: a review and protocol for perinatal management. Obst Gynecol 1983;62:539-549.

3. Schrander-Srumpel C, Fryns JP. Congenital hydrocephalus: nosology and guidelines for clinical approach and genetic counseling. Eur J Pediatr 1998;157:355-362.

4. Cavalcanti DP, Salomão MA. Incidência de hidrocefalia congênita e o papel do diagnóstico pré-natal. J Pediat; 2003;79:135-140.

5. van der Put NMJ, van Sreaaten HWM, Trijbels FJM, Blom HJ. Folate, homocysteine and neural tube defects: an overview. Exp Biol Med (Maywood) 2001;226:243-70.

6. Zlotogora J, Sagi M, Cohen T. Familial hydrocephalus of prenatal onset. Am J Med Genet; 1994;49:202-204.

7. Fryns JP, Spaepen A, Cassiman JJ, van den Berghe H. X linked complicated spastic paraplegia, MASA syndrome and $X$ linked hydrocephalus owing to congenital stenosis of the aqueduct of Sylvius: variable expression of the same mutation at Xq28. J Med Genet 1991;28:429-432.

8. Online Mendelian Inheritance in man (TM). Johns Hopkins University, Baltimore, MD. MIN Number *236680: 2001: Word Wide Web URL: http: / / www.ncbi.nlm.nih.gov/omim/

9. Strain L, Gosden CM, Brock DJH, Bonthron DT. Genetic heterogeneity in X-linked hydrocephalus: linkage to markers within Xq27.3. Am J Med Genet 1994:54:236-243.

10. Fransen E, van Camp G, D’Hooge R, Vits L, Willems PJ. Genotypephenotype correlation in L1 associated diseases. J Med Genet 1998;35:399404.

11. Fryns JP, Schander-Stumpel C, De Die-Smuldeers C, Borghgraef M, van dedr Berghe H. MASA syndrome: delineation of the clinical spectrum at prepubertal age. Am J Med Genet 1992;43:402-407.

12. Rietschel M, Friedl W, Uhlhaas S, Neugebauer M, Heimann D, Zerres K. MASA syndrome: clinical variability and linkage analysis. Am J Med Genet 1991;41:10-14.

13. Kaepernick L, Legius E, Higgins J, Kapru S. Clinical aspects of the MASA syndrome in a large family, including expressing females. Clin Genet 1994;45:181-185.

14. Bennet RL, Steinhaus KA, Uhrich SB, et al. Recommendations for standardized human pedigree nomenclature. Am J Med Genet 1995;56:745-752.

15. Smith DW, Clarren SK, Harvey MA. Hyperthermia as a possible teratogenic agent. J Pediatr 1978;92:878-883.

16. Layde PM, Edmonds LD, Erickson JD. Maternal fever and neural tube defects. Teratology 1980;21:105-108.

17. Pleet H, Graham JMJr, Smith DW. Central nervous system and facial defects associated with maternal hyperthermia at four to 14 weeks' gestation. Pediatrics 1981;67:785-789.

18. Upfold JB, Smith MS. Maternal hyperthermia as a cause of idiopathic mental retardation. Med Hypotheses 1988;27:89-92.

19. Polivka BJ, Nickel JT, Wilkins JP 3rd Urinary tract infection during pregnancy: a risk factor for cerebral palsy? J Obstet Gynecol Neonatal Nurs 1997;26:405-413

20. McDermott S, Callaghan W, Szwejbka L, Mann H, Daguise V. Urinary tract infections during pregnancy and mental retardation and developmental delay. Obstet. Gynecol 2000;96:113-119.

21. McDermott S, Daguise V, Mann H, Szwejbka L, Callaghan W. Perinatal risk for mortality and mental retardation associated with maternal urinary-tract infections. J Fam Pract 2001;50:433-437.

22. Quan L, Smith DW. The Vater association, vertebral defects, anal atresia, T-E fistula with esophageal atresia, radial and renal dysplasia: a spectrum of associated defects. J Pediatr 1973;82:104.

23. Sujansky E, Leonard B. VACTERL association with hydrocephalus: a new recessive syndrome? Am J Hum Genet 1983:35:119A.

24. Briad ML, Le Mener, Plauchu H et al. Association VACTERL et hydrocephalie: une nouvelle entite familiale. Ann. Genet 1984;27:220-223.

25. Wang H, Hunter AGW, Clifford B, McLaughlin M, Thompson D. VACTERL with hydrocephalus: spontaneous chromosome breakage and rearrangement in a family showing apparent sex-linked inheritance. Am J Med Genet 1983;47:114-117.

26. Froster UG, Wallner SJ, Reusche E, Schwinger E, Rehder. VACTERL with hydrocephalus and branchial arch defects: prenatal, clinical, and autopsy findings in two brothers. Am J Med Genet 1996;62:169-172.

27. Lomas FE, Dahlstrom JE, Ford JH. VACTERL with hydrocephalus: family with X-Linked VACTERL-H. Am J Med Genet 1998;76:74-78.

28. Halladay J, Chow CW, Wallace D, Danks DM. X linked hydrocephalus: a survey of a 20 year period in Victoria, Australia. J Med Genet 1986; 23:23-31.

29. Verhagen WIM, Bartels, RHAM, Fransen E, van Camp G, Renier WO, Grotenhuis JA. Familial congenital hydrocephalus and aqueduct stenosis with probably autosomal dominant inheritance and variable expression. J Neurol Sci; 1998;158:101-105.

30. Vincent $C$, Kalatzis V, Compain S et al. A proposed new contiguous gene syndrome on $8 \mathrm{q}$ consists of Branchio-Oto-Renal (BOR) syndrome, Duane syndrome, a dominant form of hydrocephalus and trapeze aplasia: implications for the mapping of the BOR gene. Hum Mol Genet 1994;3:1859-1866.

31. Kaplan P. X-linked recessive inheritance of agenesis of the corpus callosum. J Med Genet 20:1983:122-124.

32. Fransen E, van Camp G, D’Hooge R, Vits L, Willems PJ Genotype-phenotype correlation in L1 associated diseases. J Med Genet 1998;35: 399404.

33. Parisi MA, Kapur RP, Neilson I et al. Hydrocephalus and intestinal aganglionosis: is L1CAM a modifier gene in Hirschsprung disease? Am J Med Genet 2002;108:51-56. 